\title{
A satisfação sexual de mulheres jovens considerando dois conceitos: excitação e orgasmo
}

\author{
Sexual satisfaction in young women considering two concepts: \\ excitation and orgasm
}

\author{
Andréia Silva Reis ${ }^{1}$; Cristiani Kobayashi ${ }^{2}$
}

Resumo

A sexualidade feminina é assunto discutido na sociedade atual, porém a satisfação sexual feminina não é comumente estudada. O objetivo deste trabalho foi avaliar a satisfação sexual de mulheres jovens de São Paulo, considerando a excitação e o orgasmo. Foi desenvolvido um estudo de campo descritivo de natureza quantiqualitativa sobre a satisfação sexual feminina. A população deste estudo foi composta por 60 mulheres com idade entre 18 e 21 anos, sexualmente ativas, aplicando-se um questionário com questões sobre sexualidade. Os resultados apontaram que as mulheres não necessitam alcançar o orgasmo para se sentirem satisfeitas sexualmente, mas ao mesmo tempo preferem alcançá-lo a ficarem extremamente excitadas, conseguiram diferenciar excitação de orgasmo, afirmaram estar mais preocupadas com o prazer do parceiro do que com o seu próprio e creditam a estes a responsabilidade sobre seu prazer. Concluímos que é importante promover outros estudos na área da sexualidade, atentando-se para o público feminino e em especial, para o público adolescente que inicia a atividade sexual cada vez mais precocemente.

Palavras-chaves: Sexualidade. Excitação. Orgasmo. Disfunções.

\begin{abstract}
Female sexuality is a topic discussed in today's society, but the assessment of female sexual satisfaction is not commonly studied. The aim of this study was to evaluate sexual satisfaction young women of St. Paul, considering arousal and orgasm. We developed a field study was a quantitative and qualitative descriptive about female sexual satisfaction. The study population consisted of 60 women aged 18 to 21 years sexually active, applying a questionnaire on sexuality. The results showed that women do not need to reach orgasm to feel sexually satisfied, but at the same time prefer to achieve it to are overly excited, managed to differentiate arousal to orgasm and said they were more concerned with the enjoyment of the partner than with your own. The nurse should know the female sexual characteristics, to improve their care, contributing to the promotion of sexual health in order to prevent further complications.
\end{abstract}

keywords: Sexuality. Excitement. Orgasm. Dysfunctions.

\footnotetext{
${ }^{1}$ Faculdade Santa Marcelina. Universidade Paulista. E-mail: andreia_silvareis@yahoo.com.br

${ }^{2}$ Psicóloga, doutora pelo Instituto de Psicologia da USP. E-mail: criskobayashi@yahoo.com.br
} 


\section{Introdução}

Asexualidade é um tema discutido com frequência, atentando-se na maioria das vezes aos jovens, que iniciam a vida sexual cada vez mais precocemente. Nas mulheres, apesar do corpo estar fisicamente pronto para reprodução, a maturação psicológica não está no mesmo nível de desenvolvimento, logo a adolescente sente-se confusa, pois a menarca é tida como um rito de passagem: a menina deixou de ser menina para torna-se uma mulher. Para muitos desenvolvimentalistas, o fim da adolescência marca o ponto no qual o indivíduo torna-se razoavelmente independente dos pais e inicia atividades como trabalho e adquirem algumas responsabilidades, logo, a adolescência funde-se à fase adulta (FRIDMAN, 2009; SHAFFER, 2008).

Segundo o Ministério da Saúde (BRASIL, 2012) o Estatuto da Criança e do Adolescente considera adolescentes indivíduos de 12 a 18 anos de idade e em alguns casos excepcionais dispostos na lei, podem-se considerar adolescentes indivíduos até os 21 anos.

A adolescência pode ser vista como uma das fases mais complexas na vida de qualquer ser humano. Esta fase é caracterizada pela existência de diversos conflitos como a busca por estabilidade financeira, emocional, e grande preocupação em encontrar parceiros sexuais, criar laços de amizade, ou seja, companheirismo, onde há maior preocupação com a sexualidade (AMERICAN PSYCHIATRIC ASSOCIATION, 2000; PRATA; DIAS, 2008).

Segundo estudo realizado pelo Ministério da Saúde (BRASIL, 2000) a idade mediana verificada para a iniciação sexual nas adolescentes de 16 a 19 anos foi de 15,0 anos, valor consideravelmente diminuído em vista ao estudo anteriormente realizado em 1984 onde a mediana foi de 16,0 anos, demonstrando assim, que a iniciação sexual nas mulheres tende a ocorrer cada vez mais cedo.

Existe uma expectativa social que a experiência sexual em mulheres jovens deva ocorrer em relacionamentos mais sérios, como por exemplo, no namoro. Para muitas jovens e seus familiares, o amor tem papel fundamental na aceitação e naturalização do sexo nos relacionamentos pré-conjugais. Percebese que, para a mulher, é como se o amor validasse o sexo. Há também o medo do abandono pelo parceiro pela falta de atividade sexual, com isso, muitas vezes acabam sentindo-se forçadas a ceder, sem realmente estarem preparadas ou desejarem (HEILBORN, 1999; TRINDADE; FERREIRA, 2008)

$\mathrm{O}$ ato sexual, além da função óbvia relacionada à reprodução da espécie, também possui outros signos como satisfação sexual, sensação de prazer e aumento de vínculos afetivo-amorosos, sendo o orgasmo o objetivo a ser alcançado por ambos os parceiros (BORGES, 2007; FERNANDES, 2012).

Segundo Kaplan (1997), Master e Johnson (1996) e Brasil (2010) temos estabelecido um novo modelo para o ciclo das respostas aos estímulos sexuais, em ambos os sexos, definindo a resposta sexual considerada saudável, em um conjunto de quatro etapas obedecidas em sequência:

1) Fase de desejo sexual: O desejo sexual pode ser definido como um conjunto de sensações extremamente específicas que o indivíduo sente e que o fazem procurar ou ser receptivo à experiência sexual. As fontes que estimulam o desejo sexual variam de pessoa para pessoa. Fatores como o estado de saúde, depressão, ansiedade, sentirse explorado de alguma forma pelo outro, medo de envolvimento afetivo, dentre outros, podem influenciar negativamente o desejo sexual.

2) Fase de excitação: Desencadeada pelo desejo é a preparação para o ato sexual. Estímulos psicológicos (pensamentos e fantasias) e/ou físicos (tato, olfato, gustação, audição e visão) podem levar à excitação. Junto com sensações de prazer, surgem alterações corporais que são representadas basicamente no homem, pela ereção e na mulher, pela vasocongestão da vagina 
e da vulva e pela lubrificação vaginal.

3) Fase de orgasmo: Considerado o clímax do prazer sexual, que ocorre após uma fase crescente de excitação. No homem, o mesmo pode ser considerado centralizado no órgão genital junto com a sensação de prazer, ocorre a ejaculação. $\mathrm{Na}$ mulher, ocorrem contrações musculares rítmicas em volta da entrada da vagina. Sinais físicos são percebidos nessa fase, em ambos os sexos, como por exemplo: taquicardia, taquipnéia, elevação da pressão arterial e sudorese.

4) Fase de resolução: Considerado o período em que o organismo retorna às condições físicas e emocionais anteriores à fase de excitação.

No orgasmo feminino, há uma grande repercussão em toda a extensão corporal, ou seja, na mulher o orgasmo não é centralizado como no homem, mas sim, generalizado e estende-se por toda genitália externa e interna, atingindo áreas de todo o corpo (ABDO; FLEURY, 2006; HITE, 1983; KAPLAN, 1997; MASTERS; JOHNSON, 1996).

No momento do orgasmo pode-se observar: contração da musculatura da vagina, elevação do útero, contração rítmica dos músculos pélvicos e do esfíncter anal, excitação mamilar, eretilidade clitoridiana, turgescência orbicular vaginal acompanhada de hiperemia e secreção humoral e de prazer excitante em toda a genitália externa e/ ou em alguns casos, de alguns pontos específicos da genitália externa e/ou interna (ABDO; FLEURY, 2006; HITE, 1983; KAPLAN, 1997; MASTERS; JOHNSON, 1996).

Os estudos direcionados a esse público que tratam da sexualidade da mulher jovem visando a satisfação sexual e a compreensão dos mecanismos femininos usados para se alcançar essa satisfação são importantes e devem ser incentivados, pois apenas o prazer sexual masculino foi sempre discutido e levado em consideração, dado o preconceito em relação à mulher que deve apenas satisfazer o homem e reproduzir. Ideologia machista e preconceituosa, porém fortemente presente no dia a dia da população de todo o mundo e em particular em nações mais ortodoxas (DOLTO, 1996; HITE, 1983; XAVIER, 2007).

É importante atentar que a saúde da mulher inclui a possibilidade do exercício de uma sexualidade plena e satisfatória. Nesse sentido, a atuação dos profissionais de saúde deve seguir os preceitos da educação em saúde, conscientizando esse público sobre os seus direitos, explicando o funcionamento de seu corpo, ajudando a identificar suas dificuldades e, investigar a origem das mesmas, contribuindo assim, para a promoção da saúde sexual dessas mulheres, a fim de evitar, complicações posteriores de cunho psicológicos, fisiológicos e sociais. (PRATA; DIAS, 2008; XAVIER, 2007).

Para a enfermagem e para demais profissionais da saúde, é relevante o conhecimento dessas características femininas, conhecendo aqueles que procuram ajuda, identificando as dificuldades existentes e desenvolvendo técnicas para lidar com as mesmas, conseguindo assim, atender às suas necessidades.

\section{Objetivo}

Avaliar a satisfação sexual de mulheres jovens entre 18 e 21 anos da cidade de São Paulo, considerando a excitação e o orgasmo.

\section{Metodologia}

Esta foi uma pesquisa de campo, descritiva de natureza quanti-qualitativa, realizada em duas universidades particulares, da cidade de São Paulo. A amostra do estudo foi composta por 60 jovens mulheres sexualmente ativas de 18 a 21 anos. Foi utilizado um questionário estruturado para a coleta de dados, com questões abertas e fechadas sobre as experiências sexuais das jovens mulheres. 
As questões fechadas foram codificadas para análise estatística realizada com o auxílio do programa STATA versão 13.1. Para os dados qualitativos utilizou-se a técnica de análise de conteúdo de Bardin (2009). As respostas das questões abertas foram reunidas em grupos temáticos conforme definição de Bardin (2009): "O tema é a unidade de significação que se liberta naturalmente de um texto analisado segundo critérios relativos à teoria que serve de guia à leitura" (p.105).

A pesquisa foi submetida à análise e apreciação do Comitê de Ética em Pesquisa com Seres Humanos da Universidade Paulista, sendo aprovada sob o Parecer CEP: $n^{\circ}$ 97.418/2012. Foram utilizados nomes fictícios para a preservação da identidade dos participantes.

\section{Resultados e Discussão}

Após a coleta de dados e análise, apresentamos a seguir os principais resultados encontrados. O primeiro tema analisado se referia à diferença entre excitação e orgasmo. As respostas sobre o que as excitavam foram agrupadas, resultando em três categorias: estímulos físicos, mentais e ambientais. Destes, o estímulo mais comentado e apontado foi o físico, como "toque", "beijos", "receber sexo oral", "masturbação realizada pelo parceiro", presente em $84 \%$ das respostas. Os estímulos mentais estavam presentes em 21,3\% das respostas, alguns destes foram: "palavras faladas ao ouvido/frase intimas", "sentir-se desejada", "proporcionar prazer ao parceiro", "fantasias". Os estímulos ambientais foram descritos em 17,3\% das respostas, como exemplos podemos citar: "bebida", "clima/filmel musica" e "em situações inusitadas".

Quando questionadas sobre o orgasmo, 95\% das mulheres referiram sensações mentais como "bem estar", "prazer extremo" e 91,8\% relataram sensações físicas como "tremor nos membros inferiores", "arrepios/calafrios", "respiração ofegante", "formigamento em todo o corpo", "contrações vaginais", "espasmos musculares". A partir da análise das respostas, conseguimos comparar com as fases de respostas sexuais humanas descritas por Kaplan (1997), Master e Johnson (1996) e notamos que a grande maioria das mulheres entrevistadas conseguiu diferenciar excitação de orgasmo.

Tabela 1 - Referente à satisfação sexual das jovens mulheres, ao orgasmo e sua excitação. São Paulo, 2012.

\begin{tabular}{l|l|l}
\hline Variável & $\mathbf{N}^{\mathbf{0}}$ & $\%$ \\
\hline Sente-se satisfeita sexualmente & & \\
\hline Sim & 53 & 88,3 \\
Não & 07 & 11,7 \\
\hline Já teve orgasmo & & \\
\hline Sim & 49 & 81,6 \\
Não & 10 & 16,7 \\
Não responderam & 01 & 1,7 \\
\hline Para se satisfazer precisa alcançar o orgasmo & 15 & 25,0 \\
\hline Sim & 41 & 68,3 \\
Não & 04 & 6,7 \\
Não responderam & \multicolumn{2}{|l}{} \\
\hline Qual escolheria, por considerar ser melhor & 30 & 50,0 \\
\hline Chegar ao orgasmo & 25 & 41,7 \\
Ficarextremamente excitada & 05 & 8,3 \\
Não responderam & $\mathbf{6 0}$ & $\mathbf{1 0 0}$ \\
\hline TOTAL & \multicolumn{2}{l}{}
\end{tabular}

Fonte: Autoria própria. 
Sobre o tema satisfação sexual, os resultados apontaram que $88,3 \%$ das mulheres entrevistadas afirmaram se sentir satisfeitas sexualmente e, cerca de $80 \%$ já haviam alcançado o orgasmo ao menos uma vez nas relações sexuais com os/ as atuais parceiros/as. Por outro lado, 68,3\% delas responderam não necessitar do orgasmo para se sentirem satisfeitas sexualmente. Essas contradições apresentadas nas respostas deixaramnos com algumas dúvidas: será que essas mulheres realmente conheciam seu corpo e conseguiam diferenciar excitação de orgasmo? Ou será que elas apenas mostraram seu conhecimento teórico sobre a sexualidade? Podemos concluir que a sexualidade não está diretamente relacionada ao orgasmo para as mulheres? Autores como Dolto (1996) e Hite (1983) apontam fatores subjetivos como, por exemplo: presença de parceiro, romance, dinâmica do casal e ou relacionamento, intensidade de relacionamento, comprometimento, respeito, dentre outros, que não o orgasmo em si, como sendo primordiais e significantes para uma parcela considerável das jovens.

Outra questão interessante foi a respeito de suas preferências, em que $50 \%$ das mulheres escolheriam ter um orgasmo a ficarem extremamente excitadas, enquanto que, por volta de $42 \%$ afirmaram o contrário, que achavam melhor ficar extremamente excitadas a ter um orgasmo. Notamos aqui, que apesar de não terem apontado o orgasmo como fator essencial no relacionamento, uma parcela considerável dessas mulheres o desejam.

Quando questionadas sobre o porquê se sentiam satisfeitas sexualmente, as respostas resultaram em duas categorias. A primeira categoria, com o maior número de mulheres, corresponde à presença de parceiro sexual e relacionamento:

Porque ele satisfaz minhas necessidades, porque me sinto à vontade com ele (MARTA).

Gosto do meu relacionamento (MARIA).
Por estar com a pessoa que eu gosto, que me faz feliz (MARCIA).

Na segunda categoria, a atribuição da satisfação sexual é dada pelo alcance do orgasmo no ato sexual, essa categoria foi composta por apenas quatro mulheres:

Porque sinto excitação em todas as relações, chego ao orgasmo na maioria (...) (MARCIA).

Porque minhas "expectativas" foram positivamente alcançadas. (MARCELA).

Porque eu sinto tesão e chego ao orgasmo na maioria das vezes. (MARA).

Analisando tais resultados podemos supor que a satisfação sexual dessas jovens não está ligada à presença do orgasmo, ou seja, o orgasmo não é sinônimo de satisfação sexual. Percebemos então que a somatória de vários aspectos que compõem um relacionamento resulta na satisfação sexual da jovem, pois tais aspectos interferem e refletem no ato sexual e na percepção que a mulher tem sobre o mesmo (DOLTO, 1996; FERNANDES, 2012; HITE, 1983; PRATA; DIAS, 2008).

No tema relacionamento afetivo, observamos que, quase $40 \%$ das mulheres, afirmaram que manteriam o relacionamento mesmo não estando satisfeitas sexualmente. Aparentemente, o que importa para essas mulheres são outros aspectos que compõem um relacionamento afetivoamoroso, e não somente o sexo. Para um melhor delineamento de quais seriam esses aspectos, mais estudos sobre o tema se fazem necessários (FERNANDES, 2012; PRATA; DIAS, 2008).

Ainda para comparar os dados e resultados, temos na tabela 2 questões relacionadas à manutenção de relacionamento sem atingir satisfação sexual e a preocupação sobre o prazer do parceiro (a) durante a relação sexual: 
Tabela 2 - Referente ao relacionamento afetivo e a satisfação sexual. São Paulo, 2012.

\begin{tabular}{l|l|c}
\hline Variável & $\mathbf{N}^{\mathbf{0}}$ & $\%$ \\
\hline \multicolumn{1}{|c}{ Manteria um relacionamento se não conseguisse atingir satisfação sexual } \\
\hline Não & 33 & 55,0 \\
Sim & 23 & 38,3 \\
Não responderam & 04 & 6,7 \\
\hline $\begin{array}{l}\text { Preocupa-se mais em dar prazer ao parceiro do que a ela mesma durante a } \\
\text { relação sexual }\end{array}$ & 30 & 50,0 \\
\hline Às vezes & 27 & 45,0 \\
Sempre & 02 & 3,3 \\
Não responderam & 01 & 1,7 \\
Nunca & $\mathbf{6 0}$ & $\mathbf{1 0 0}$ \\
\hline TOTAL
\end{tabular}

Fonte: Autoria própria.

O que mais surpreende é perceber que, em 95\% das respostas as jovens afirmam estar mais preocupadas com o prazer sexual do parceiro. Esse dado revela o quanto essas jovens estão voltadas para a satisfação do outro, de modo que a responsabilidade pelo prazer alheio é delas e o seu próprio prazer sexual é de responsabilidade do(a) parceiro(a). Observamos aqui uma inversão de responsabilidades que as mulheres fazem quase que imperceptivelmente. (DOLTO, 1996; FERNANDES, 2012).

Atualmente, as mulheres defendem a liberdade de expressão e escolha, a liberdade sexual, como escolher o parceiro sexual e qual o nível de envolvimento que terão. Apesar das intensas manifestações, lutas, protestos, conflitos e busca por liberdade de expressão e direito sobre seu próprio corpo, percebemos que discursar é diferente de agir e no caso da sexualidade feminina, quanto ao seu prazer e a relação entre seu corpo, seu prazer e o envolvimento com o outro, percebe-se que as mulheres ainda não conseguiram libertar-se das amarras psicológicas e morais impostas pela sociedade ao longo desses anos (LARA et al. 2008; PABLO; SOARES, 2004; XAVIER, 2007).

\section{Considerações Finais}

As mulheres são complexas em sua totalidade e, em especial, na questão de sua sexualidade. O presente estudo trouxe indícios de que, as mulheres jovens do século XXI, apesar das lutas e discursos sobre independência e liberdade sexual, ainda estão em busca de um relacionamento e não apenas de satisfação sexual. Para elas, o envolvimento afetivo com o(a) parceiro(a) é mais importante do que o ato sexual em si. Aparentemente, estas mulheres ainda estão presas aos tabus sociais em relação ao sexo e ao desejo de estabelecer um relacionamento romântico e duradouro.

Com esta pesquisa muitas perguntas surgiram e esperamos ter contribuído não apenas para respondê-las, mas para aguçar o interesse de outros pesquisadores para que mais pesquisas nesta área sejam realizadas.

\section{Referências}

ABDO, C.; FLEURY, H. Aspectos diagnósticos e terapêuticos das disfunções sexuais femininas. Revista de Psiquiatria Clínica, São Paulo, v. 33, n. 3, p. 162-167, 2006. 
AMERICAN PSYCHIATRIC ASSOCIATION. Manual diagnóstico e estatístico de transtornos mentais. 4. ed. Porto Alegre: ARTMED, 2000.

BARDIN, L. Análise de conteúdo. Lisboa: Ed. 70, 2009.

BORGES, A. Relações de gênero e iniciação sexual de mulheres adolescentes. Revista Escola de Enfermagem [online], São Paulo, v. 41, n. 4, p. 597604, 2007.

BRASIL. Ministério da Saúde. Coordenação Nacional DST/AIDS. Centro Brasileiro de Análise e Planejamento (CEBRAP). Pesquisa sobre comportamento sexual da população brasileira e percepções sobre HIV/AIDS. Brasília, 2000.

. Ministério da Saúde. Secretaria de Atenção à Saúde. Departamento de Atenção Básica. Saúde sexual e saúde reprodutiva. Brasília, 2010.

- Secretaria Nacional de Promoção dos Direitos da Criança e do Adolescente. Estatuto da criança e do adolescente. Lei Federal $n^{\circ} 8069$, de 13 de julho de 1990. Brasília, 2012.

DOLTO, F. Sexualidade feminina: libido, erotismo, frigidez. 3. ed. São Paulo: Martins Fontes, 1996.

FERNANDES, V. Mulher rima com dor? Algumas considerações sobre a nação do prazer "masculino" e do "sofrimento feminino". Revista Latinoamericana de Geografia e Gênero, Ponta Grossa, v. 3, n. 1, p. 85-93, Jan./jul. 2012.

FRIDMAN, C. El sexo oral entre adolescentes de La cuidad de Buenos Aires y El conurbano bonarense Argentina. Revista Brasileira de Sexualidade Humana, São Paulo, v. 20, n. 1, p. 53-57, 2009.

HEILBORN, M. L. Construção de si, gênero e sexualidade. In: HEILBORN, M. L. (Org.). Sexualidade: o olhar das ciências sociais. Rio de Janeiro: J. Zahar. 1999. p. 40-58.

HITE, S. O relatório Hite: um profundo estudo sobre a sexualidade feminina. 15. ed. São Paulo: Difel, 1983.
KAPLAN, H. A nova terapia do sexo. 3. ed. Rio de Janeiro: Nova Fronteira, 1997.

LARA, L. et al. Abordagem das disfunções sexuais femininas. Revista Brasileira de Ginecologia e Obstetrícia, Rio de Janeiro, v. 30, n. 6, jun. 2008.

MASTERS, W.; JOHNSON, V. Human sexual response. Boston: Lippincott Williams and Wilkins, 1996.

PABLO, C.; SOARES, C. As disfunções sexuais femininas. Revista Portuguesa de Clínica Geral, Lisboa, v. 20, p. 357-370, 2004.

PRATA, K.; DIAS, C. O Orgasmo na vida sexual da mulher contemporânea. Revista Brasileira de Sexualidade Humana, São Paulo, v. 19, v. 1, p. 1820, jan./jul, 2008.

SHAFFER, D. Psicologia do desenvolvimento: infância e adolescência. 6. ed. São Paulo: Cengage Learning, 2008.

TRINDADE, W.; FERREIRA, M. Sexualidade feminina: questões do cotidiano das mulheres. Texto \& Contexto Enfermagem [online], Florianópolis, v. 17, n. 3, p. 417-426, 2008.

XAVIER, C. A Sexualidade feminina entre práticas divisora: da mulher "bela adormecida": sexualidade à multiorgástica: imprensa feminina e discursos de professoras. In: REUNIÃO ANUAL DA ASSOCIAÇÃO NACIONAL DE PÓSGRADUAÇÃO E PESQUISA EM EDUCAÇÃO, 30., 2007, Caxambu. Trabalhos GT23: Gênero, Sexualidade e Educação. Caxambu: ANPEd, 2007. GT23-3297, p. 1-15. 
